\title{
Distribución de alimentos de los comités de vaso de leche: programa social desarrollado por la municipalidad provincial de San Martín
}

\section{RESUMEN}

Luis Alberto Saavedra García lsaavedraga@ucvvirtual.edu.pe

\author{
Dr. Keller Sánchez Dávila \\ ksanchezd@ucv.edu.pe \\ Universidad César Vallejo
}

La investigación se planteó con el fin de diseñar una propuesta de modelo estratégico que contribuya a la distribución de alimentos de los comités de vaso de leche de la municipalidad provincial de San Martín, 2021. La investigación fue de tipo básico, con un diseño descriptivo propositivo. Existen procesos de toma de decisiones estratégicas que, de acuerdo a la teoría del procesamiento de información organizacional, la visión de la empresa permite argumentar la racionalidad procedimental y politización como un efecto negativo en la internacionalización de las pymes. Se evidenció la importancia que tiene la inteligencia estratégica de los líderes empresariales para el desarrollo y crecimiento de su organización a su cargo. Se demostró que la globalización de la producción de los alimentos influye en la estructura de la distribución de las cadenas de suministros de alimentos. en modelización de un sistema de distribución de la cadena de suministro de cereales alimentarios sostenible, mencionaron que la demanda de los alimentos, la degradación ambiental, las perdidas postcosecha y la escasez de recursos alientan a los tomadores de decisiones de los países en desarrollo a integrar los aspectos económicos y ambientales en el diseño de la red de distribución de los alimentos. El problema más importante de la competitividad de las universidades rusas es la falta de flexibilidad estratégica, que afecta a todo el sistema de educación universitaria para Rusia. Concluyendo que el modelo estratégico es una herramienta poderosa en la gestión de programas sociales que permite el cumplimiento de los objetivos estratégicos de cualquier tipo de organización de una manera eficiente; además que la distribución de los alimentos, cumple una función principal en la generación de desarrollo para la sociedad y el modelo estratégico cumple un papel importante en la determinación de la distribución de alimentos.

Palabras clave: distribución de alimentos; vaso de leche; programa social; estrategia; globalización. 


\title{
Food distribution of the milk glass committees: social program developed by the provincial municipality of San Martín
}

\begin{abstract}
The research was proposed in order to design a proposal for a strategic model that contributes to the distribution of food by the glass of milk committees of the provincial municipality of San Martín, 2021. The research was of a basic type, with a descriptive and purposeful design - There are strategic decision-making processes that, according to the theory of organizational information processing, the vision of the company allows arguing procedural rationality and politicization as a negative effect on the internationalization of SMEs. The importance of the strategic intelligence of business leaders for the development and growth of the organization under their charge was evidenced. The globalization of food production was shown to influence the distribution structure of food supply chains. in modeling a sustainable food grain supply chain distribution system, they mentioned that food demand, environmental degradation, post-harvest losses and scarcity of resources encourage decision-makers in developing countries to integrate economic and environmental aspects in the design of the food distribution network. The most important problem of the competitiveness of Russian universities is the lack of strategic flexibility, which affects the entire university education system for Russia. Concluding that the strategic model is a powerful tool in the management of social programs that allows the fulfillment of the strategic objectives of any type of organization in an efficient way; In addition to the distribution of food, it plays a main role in generating development for society and the strategic model plays an important role in determining the distribution of food.
\end{abstract}

Keywords: food distribution; glass of milk; social program; strategy; globalization.

Artículo recibido: 18. Julio. 2021

Aceptado para publicación: 08. Agosto. 2021 Correspondencia: lsaavedraga@ucvvirtual.edu.pe

Conflictos de Interés: Ninguna que declarar

\section{INTRODUCCIÓN}


El hambre afecta a 42,5 millones de personas en América Latina y el Caribe, y se dirige al 6,5\% de la población provincial. En América Latina y el Caribe, las tasas de desnutrición se han expandido en los últimos tiempos, generalmente como resultado de la circunstancia en América del Sur, donde el nivel de personas hambrientas se expandió de 4.6\% en 2013 a $5.5 \%$ en 2018, siendo quizás las naciones más influenciadas. Venezuela (Organización de las Naciones Unidas para la Agricultura y la Alimentación [FAO], 2019). La ausencia de alimentos es un tema que se viene planteando desde hace bastante tiempo, razón por la cual en 1997 en México el Programa de Capacitación, Bienestar y Sustento (PROGRESA) se enfocó plenamente en atender las diversas causas de la indigencia.

De igual manera, se realizaron diferentes proyectos con interés específico, por ejemplo, los programas escolares de vaso de leche, los cuales se han desplegado para afectar bien el estado nutricional y el avance de los niños, sumándose a su giro de eventos (Esquivel, 2018, p. 13). El creador mencionado anteriormente hace referencia a que estos proyectos ayudan a cumplir con los objetivos habituales, combatiendo la insalubridad, desarrollando aún más la participación escolar, mejorando la ejecución académica, potenciando la zona lechera y aligerando el número de habitantes en situación económica problemática, para vencer la inestabilidad alimentaria donde se encuentran. Su objetivo definitivo es nivelar su sanación $y$, en consecuencia, contribuir al trabajo en la satisfacción personal.

Estos Programas de Apoyo Social Alimentario en Perú, entre ellos Qali Warma, el programa Vaso de Leche (PVL), el Programa Cuidando Escolares (PAE) y los Comedores Sociales; queman una pieza colosal del plan financiero estatal. Para la PVL, el estado dispensa alrededor de US \$ 95 millones para atender a los destinatarios: niños de 0 a 6 años, madres embarazadas y lactantes, así como niños de 7 a 13 años, ancianos y afectados por la tuberculosis con una ración diaria de alimentos de leche o alimentos comparables, sin embargo, hasta la fecha su compromiso de trabajar en la satisfacción personal de los destinatarios es oscuro, aunque el programa ha existido durante mucho tiempo, en realidad tiene deficiencias en la programación del transporte de los insumos y el proceso de obtención (Antón, 2016, p. 14).

Si bien este programa social tiene una estructura legítima de relacionamiento, con sistemas y otros, generalmente no se satisfacen por deficiencias o olvidos en la interacción de ejecución. A partir de las evaluaciones realizadas en mejores lugares del programa del vaso de leche, se descubren dos componentes repetidos: los datos no son valiosos ni convenientes 
y el enfoque de los destinatarios generalmente no incluye a todas las personas con salarios bajos o personas con deficiencias dietéticas, mutilando la razón del Programa (Neira, 2020, p.14). Siendo el programa del vaso de leche (PVL), uno de los pocos proyectos que tiene inclusión pública, ya que hay alrededor de 1834 distritos en todo el país, que dan una distribución diaria de alimentos a una población débil que se enfrenta a la incertidumbre alimentaria y que su condición monetaria no es adecuada para cumplir con estos requisitos esenciales.

Un informe de la Contraloría General de la República sobre el examen de responsabilidad muestra que el gasto es ejecutado todos los años por todas las regiones y lugares comunes, sin embargo, no hay sustancia que en consistencia con sus capacidades deba controlar, planificar, filtrar, preparar. y evaluar el efecto que produce la ejecución del uso entre la población receptora y los objetivos del programa, el cual debe estar firmemente conectado con el aspecto nutricional y focalizado de la población beneficiada (Antón, 2016, p.16).

El programa del vaso de leche (PVL) que gestiona la Municipalidad Provincial de San Martín se presenta con la misión de atender el tema alimenticio en poblaciones vulnerables y pobres. Este programa tiene un aproximado 73 OBS (Organizaciones Sociales de Base) entre comités y clubes de madres, llegando a atender a por lo menos 4873 beneficiarios distribuidos en la ciudad de Tarapoto. Sin embargo, en diversos sitios tales como en zonas rurales lejanas y de difícil acceso y que también se llevan a cabo este tipo de programa social presentan diversos problemas tales como la demora en la entrega de productos, afectando la ración diaria del poblador beneficiaria de este programa social, al no cumplirse con el cronograma establecido. Asimismo, existe una gestión inadecuada por parte de los funcionarios municipales, la cual fue reflejado en la adquisición de los productos por la espera de la formalización del contrato con las empresas proveedoras para iniciar la distribución de los mismos. Por otro lado, es importante señalar que este programa social puede estar amenazado con el recorte del presupuesto asignado por parte de los entes gubernamentales, esto debido a diversos factores como la transparencia de los gastos realizados, problemas de organización, selección dudosa a empresas proveedoras, la calidad de los productos adquiridos del programa social, información no clara e imprecisa de la selección de beneficiarios que muchas veces no son a lo estipulado, y que han puesto en evidencia una situación problemática que cuestiona el direccionamiento de este programa social. 


\section{ESTRATEGIAS METODOLÓGICAS O MATERIALES Y MÉTODOS}

\section{Diseño de estudio}

Corresponde al tipo básico, la cual se caracteriza por la inexistencia de manipulación de variables, enfocándose en la descripción de los hechos y fenómenos en su estado natural (Hernández et al., 2014).

Pertenece al diseño descriptivo propositivo. Este diseño se caracteriza por tener el siguiente esquema:

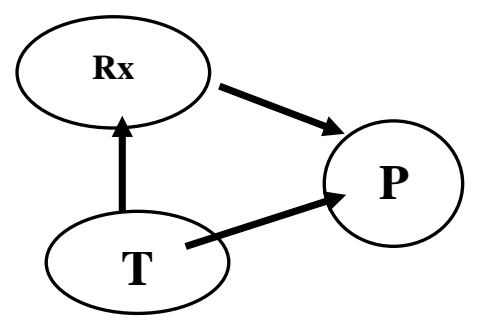

\section{Dónde:}

Rx : Diagnóstico de la realidad de la distribución de alimentos de los comités de vaso de leche de la municipalidad provincial de San Martín, 2021.

T : Estudios teóricos.

P : Propuesta validada.

\section{Procedimiento de recolección de datos}

Según la finalidad del estudio de diseñar una propuesta de modelo estratégico para la distribución de alimentos de los comités de vaso de leche, se solicitará permiso a las autoridades respectivas del programa y de la municipalidad provincial de San Martín. Mediante la autorización y el consentimiento de su participación se procederá a aplicar una encuesta con la finalidad de recolectar la información brindada por los participantes.

\section{Técnicas de análisis de datos}

Después de la recolección de los datos brindada por los participantes, estas serán digitalizadas para ser procesadas mediante software estadístico. Mediante la estadística descriptiva analizaremos la situación actual de la distribución de los comités de vaso de leche y en base a ello se diseñará un modelo estratégico para la distribución de alimentos.

\section{RESULTADOS.}


Los resultados se realizaron mediante tablas de análisis, teniendo en cuenta artículos de revistas indexadas a nivel internacional y nacional referente a las variables de estudio de la siguiente forma: 


\subsection{Estudios sobre modelos estratégico}

\section{DATOS}

\begin{tabular}{|c|c|c|c|c|c|}
\hline 1. Autor & Año & \multicolumn{2}{|c|}{ Nombre } & Revista & Vol. y núm. \\
\hline $\begin{array}{l}\text { Petrou, Andreas P. } \\
\text { Hadjielias, Elias } \\
\text { Thanos, Ioannis C. } \\
\text { Dimitratos, Pavlos }\end{array}$ & (2020) & \multicolumn{2}{|c|}{$\begin{array}{c}\text { Strategic decision-making } \\
\text { processes, international } \\
\text { environmental munificence } \\
\text { and the accelerated } \\
\text { internationalization of SMEs }\end{array}$} & $\begin{array}{l}\text { International Business Review } \\
\frac{\text { https://doi.org/10.1016/j.ibusrev.20 }}{\underline{20.101735}}\end{array}$ & $25(5)$ \\
\hline \multicolumn{6}{|c|}{ CONTENIDO } \\
\hline Tipo y Diseño & $\begin{array}{l}\text { Población y } \\
\text { Muestra }\end{array}$ & Instrumentos & Aspectos éticos & Resultados & Conclusión \\
\hline $\begin{array}{l}\text { Estudio de tipo } \\
\text { básica y diseño no } \\
\text { experimental }\end{array}$ & 176 pymes & $\begin{array}{l}\text { Entrevista y } \\
\text { análisis } \\
\text { documental }\end{array}$ & $\begin{array}{l}\text { Presentación de } \\
\text { información } \\
\text { objetiva. }\end{array}$ & $\begin{array}{l}\text { La racionalidad procesal y la } \\
\text { politización de las pymes reducen la } \\
\text { probabilidad } \quad \text { de } \\
\text { internacionalización acelerada }\end{array}$ & $\begin{array}{l}\text { Estas relaciones son más } \\
\text { pronunciadas en entornos } \\
\text { internacionales generosos, lo que } \\
\text { indica que cuando la } \\
\text { internacionalización acelerada se ve } \\
\text { limitada por procesos de toma de } \\
\text { decisiones estratégicas, las pymes } \\
\text { pueden perder oportunidades en } \\
\text { mercados internacionales atractivos }\end{array}$ \\
\hline
\end{tabular}




\begin{tabular}{|c|c|c|c|c|c|}
\hline \multicolumn{6}{|c|}{ DATOS } \\
\hline 2. Autor & Año & \multicolumn{2}{|c|}{ Nombre } & Revista & Vol. y núm. \\
\hline $\begin{array}{l}\text { Guraou, Caolin } \\
\text { Duquesnois, } \\
\text { Franck } \\
\text { Catanzaro, } \\
\text { Alexis }\end{array}$ & $(2017)$ & \multicolumn{2}{|c|}{$\begin{array}{l}\text { Entrepreneurial orientation, } \\
\text { strategic processes and SMEs' } \\
\text { performance in a turbulent } \\
\text { environment: an investigation of } \\
\text { the Languedoc-Roussillon wine } \\
\text { sector }\end{array}$} & $\begin{array}{l}\text { International Journal of Entrepreneurship and Small } \\
\text { Business } \\
\text { https://doi.org/10.1504/IJESB.2017.085991 }\end{array}$ & $32(1 / 2)$ \\
\hline \multicolumn{6}{|c|}{ CONTENIDO } \\
\hline Tipo y Diseño & $\begin{array}{l}\text { Población } \\
\text { y Muestra }\end{array}$ & Instrumentos & Aspectos éticos & Resultados & Conclusión \\
\hline $\begin{array}{l}\text { Estudio de tipo } \\
\text { básica y diseño } \\
\text { no experimental }\end{array}$ & 160 pymes & $\begin{array}{c}\text { Entrevista y } \\
\text { análisis } \\
\text { documental }\end{array}$ & $\begin{array}{l}\text { Presentación de } \\
\text { información } \\
\text { objetiva. }\end{array}$ & $\begin{array}{l}\text { un modelo causal fuerte, que integra secuencialmente } \\
\text { las dimensiones de orientación empresarial, } \\
\text { recopilación de información, planificación } \\
\text { estratégica y desempeño empresarial. }\end{array}$ & $\begin{array}{l}\text { Las disciplinas de } \\
\text { emprendimiento } \mathrm{y} \\
\text { mercadotecnia desde } \\
\text { varios años atrás se han } \\
\text { vinculado tanto en lo } \\
\begin{array}{l}\text { conceptual como en lo } \\
\text { práctico. }\end{array}\end{array}$ \\
\hline
\end{tabular}

Ciencia Latina Revista Científica Multidisciplinar, Ciudad de México, México. ISN 2707-2207 / ISSN 2707-2215 (en línea), julio-agosto, 2021, Volumen 5, Número 4. https://doi.org/10.37811/cl rcm.v5i4.774 p.6364 


\section{DATOS}

\begin{tabular}{|c|c|c|c|c|}
\hline 3. Autor & Año & Nombre & Revista & Vol. y núm. \\
\hline $\begin{array}{l}\text { Secundo, Giustina } \\
\text { Schiuma, } \\
\text { Giovanni Jones, } \\
\text { Paul }\end{array}$ & (2019) & $6 \begin{array}{l}\text { Strategic knowledge } \\
\text { management models and } \\
\text { tools for entrepreneurial } \\
\text { universities }\end{array}$ & $\begin{array}{c}\text { Management Decision } \\
\underline{\text { https://doi.org/10.1108/MD-12-2019- }} \\
\underline{027}\end{array}$ & $57(12)$ \\
\hline
\end{tabular}

\begin{tabular}{|c|c|c|c|c|c|}
\hline Tipo y Diseño & $\begin{array}{l}\text { Población } \\
\text { y Muestra }\end{array}$ & Instrumentos & $\begin{array}{l}\text { Aspectos } \\
\text { éticos }\end{array}$ & Resultados & Conclusión \\
\hline $\begin{array}{l}\text { Básica y no } \\
\text { experimental }\end{array}$ & $\begin{array}{c}8 \text { oficinas } \\
\text { de apoyo a } \\
\text { la } \\
\text { propiedad } \\
\text { industrial. }\end{array}$ & $\begin{array}{l}\text { Entrevista y } \\
\text { análisis } \\
\text { documental }\end{array}$ & $\begin{array}{c}\text { Presentación } \\
\text { de } \\
\text { información } \\
\text { objetiva. }\end{array}$ & $\begin{array}{l}\text { Se han identificado cuatro áreas } \\
\text { principales de investigación de } \\
\text { especialización dentro del debate } \\
\text { científico; categorizamos las principales } \\
\text { áreas en: creación de conocimiento en } \\
\text { UIC; Estrategias y Modelos de } \\
\text { Conocimiento para la Universidad } \\
\text { Emprendedora; } \\
\text { emprendedora en el contexto } \\
\text { universitario; y procesos de GC para } \\
\text { incubadoras de empresas y OTT de } \\
\text { universidades }\end{array}$ & $\begin{array}{l}\text { El análisis de los trabajos incluidos en estas } \\
\text { áreas permitió derivar una conciencia más } \\
\text { sólida sobre el estado del arte de la GC y la } \\
\text { Universidad Emprendedora en términos de una } \\
\text { orientación emprendedora más consciente de } \\
\text { las personas, profesores y estudiantes, gerente } \\
\text { de la Universidad Emprendedora, Procesos de } \\
\text { GC en base a los modelos organizacionales de } \\
\text { la Universidad Emprendedora y finalmente } \\
\text { procesos de GC para la valorización de los } \\
\text { procesos de conocimiento con las incubadoras } \\
\text { y OTT de la Universidad. }\end{array}$ \\
\hline
\end{tabular}




\begin{tabular}{|c|c|c|c|c|c|}
\hline \multicolumn{6}{|c|}{ DATOS } \\
\hline 4. Autor & Año & \multicolumn{2}{|c|}{ Nombre } & Revista & Vol. y núm. \\
\hline $\begin{array}{l}\text { Kumar, V. } \\
\text { Lahiri, Avishek } \\
\text { Dogan, Orhan. }\end{array}$ & (2018) & \multicolumn{2}{|c|}{$\begin{array}{l}\text { A strategic framework for a } \\
\text { profitable business model in } \\
\text { the sharing economy }\end{array}$} & $\begin{array}{l}\text { Industrial Marketing Management } \\
\text { https://doi.org/10.1016/j.indmarman.2017.08.021 }\end{array}$ & 69 \\
\hline \multicolumn{6}{|c|}{ CONTENIDO } \\
\hline Tipo y Diseño & $\begin{array}{c}\text { Población y } \\
\text { Muestra }\end{array}$ & Instrumentos & $\begin{array}{c}\text { Aspectos } \\
\text { éticos }\end{array}$ & Resultados & Conclusión \\
\hline $\begin{array}{l}\text { Básica y no } \\
\text { experimental }\end{array}$ & $\begin{array}{l}8 \text { oficinas } \\
\text { de apoyo a } \\
\text { la propiedad } \\
\text { industrial. }\end{array}$ & $\begin{array}{l}\text { Entrevista y } \\
\text { análisis } \\
\text { documental }\end{array}$ & $\begin{array}{l}\text { Presentación } \\
\text { de } \\
\text { información } \\
\text { objetiva. }\end{array}$ & $\begin{array}{l}\text { Los servicios de economía colaborativa son adoptados } \\
\text { principalmente por la Generación Y, mientras que otras } \\
\text { generaciones aún se encuentran en la fase inicial de } \\
\text { adopción. Además, la rotación de clientes y proveedores de } \\
\text { servicios es alta. }\end{array}$ & $\begin{array}{l}\text { Argumentamos que este } \\
\text { marco de relación con el } \\
\text { cliente de doble cara } \\
\text { ayudará a las empresas a } \\
\text { tomar las medidas } \\
\text { adecuadas para mantener a } \\
\text { todos los actores } \\
\text { involucrados en el proceso } \\
\text { satisfechos, leales y } \\
\text { rentables a largo plazo }\end{array}$ \\
\hline
\end{tabular}

Ciencia Latina Revista Científica Multidisciplinar, Ciudad de México, México. ISN 2707-2207 / ISSN 2707-2215 (en línea), julio-agosto, 2021, Volumen 5, Número 4. https://doi.org/10.37811/cl rcm.v5i4.774 p.6366 


\begin{tabular}{|c|c|c|c|c|c|}
\hline \multicolumn{6}{|c|}{ DATOS } \\
\hline 5. Autor & Año & \multicolumn{2}{|c|}{ Nombre } & Revista & Vol. y núm. \\
\hline $\begin{array}{l}\text { Ahmadi, Masoud } \\
\text { Baei, Fahimeh } \\
\text { Hosseini-Amiri, Seyyed } \\
\text { Moarefi, Alireza } \\
\text { Suifan, Taghrid S. } \\
\text { Sweis, Rateb }\end{array}$ & (2020) & $\begin{array}{l}\text { Proposing } \\
\text { manager's } \\
\text { intelligence, } \\
\text { developmen } \\
\text { entrepreneu } \\
\text { organization }\end{array}$ & $\begin{array}{r}\text { model of } \\
\text { strategic } \\
\text { organization } \\
\text { and } \\
\text { behavior in }\end{array}$ & $\begin{array}{l}\begin{array}{l}\text { Journal of Management Development } \\
\text { https://doi.org/10.1108/JMD-11-2018- }\end{array} \\
\underline{0317}\end{array}$ & $39(4)$ \\
\hline \multicolumn{6}{|c|}{ CONTENIDO } \\
\hline Tipo y Diseño & $\begin{array}{l}\text { Población y } \\
\text { Muestra }\end{array}$ & Instrumentos & Aspectos éticos & Resultados & Conclusión \\
\hline $\begin{array}{l}\text { Básica y no } \\
\text { experimental }\end{array}$ & $\begin{array}{c}274 \text { miembros } \\
\text { de personal }\end{array}$ & $\begin{array}{l}\text { Entrevista y } \\
\text { análisis } \\
\text { documental }\end{array}$ & $\begin{array}{l}\text { Presentación de } \\
\text { información } \\
\text { objetiva. }\end{array}$ & $\begin{array}{l}\text { El examen de las variables del moderador, } \\
\text { incluidas las características demográficas, } \\
\text { reveló que las características demográficas } \\
\text { no tienen ningún efecto sobre la relación } \\
\text { SI-OD del gerente y la relación IS-EB del } \\
\text { gerente. Solo el género influye en la } \\
\text { relación SI-EB del gerente. }\end{array}$ & $\begin{array}{l}\text { Existe una conexión } \\
\text { positiva crítica entre la IS } \\
\text { del director y el giro } \\
\text { jerárquico de los } \\
\text { acontecimientos. Además, } \\
\text { existe una gran conexión } \\
\text { positiva entre el SI del } \\
\text { administrador y la } \\
\text { conducta empresarial. }\end{array}$ \\
\hline
\end{tabular}

Ciencia Latina Revista Científica Multidisciplinar, Ciudad de México, México. ISN 2707-2207 / ISSN 2707-2215 (en línea), julio-agosto, 2021, Volumen 5, Número 4. https://doi.org/10.37811/cl rcm.v5i4.774 p.6367 


\begin{tabular}{|c|c|c|c|c|c|}
\hline \multicolumn{6}{|c|}{ DATOS } \\
\hline 6. Autor & Año & \multicolumn{2}{|c|}{ Nombre } & Revista & Vol. y núm. \\
\hline $\begin{array}{l}\text { Parakhina, Valentina } \\
\text { Godina, Olga } \\
\text { Boris, Olga } \\
\text { Ushvitsky, Lev }\end{array}$ & (2017) & \multicolumn{2}{|c|}{$\begin{array}{l}\text { Strategic management in } \\
\text { universities as a factor of their } \\
\text { global competitiveness }\end{array}$} & $\begin{array}{l}\begin{array}{l}\text { International Journal of Educational } \\
\text { Management }\end{array} \\
\underline{\text { https://doi.org/10.1108/IJEM-03- }} \\
\underline{\underline{2016-0053}}\end{array}$ & $31(1)$ \\
\hline \multicolumn{6}{|c|}{ CONTENIDO } \\
\hline Tipo y Diseño & $\begin{array}{l}\text { Población y } \\
\text { Muestra }\end{array}$ & Instrumentos & Aspectos éticos & Resultados & Conclusión \\
\hline $\begin{array}{l}\text { Básica y no } \\
\text { experimental }\end{array}$ & $\begin{array}{c}16 \\
\text { universidades }\end{array}$ & $\begin{array}{l}\text { Análisis de } \\
\text { contenido } \\
\text { Evaluación de } \\
\text { expertos }\end{array}$ & $\begin{array}{l}\text { Presentación de } \\
\text { información } \\
\text { objetiva. }\end{array}$ & $\begin{array}{l}\text { El modelo de administración actual de } \\
\text { la mayoría de las universidades rusas } \\
\text { muestra un bajo grado de } \\
\text { productividad. }\end{array}$ & $\begin{array}{l}\text { el problema más importante de la } \\
\text { competitividad de las universidades } \\
\text { rusas es la falta de flexibilidad } \\
\text { estratégica. Podemos decir que hay } \\
\text { un carácter estructural de la crisis de } \\
\text { gestión que afecta a todo el sistema } \\
\text { de educación universitaria. }\end{array}$ \\
\hline
\end{tabular}

Ciencia Latina Revista Científica Multidisciplinar, Ciudad de México, México. ISN 2707-2207 / ISSN 2707-2215 (en línea), julio-agosto, 2021, Volumen 5, Número 4. https://doi.org/10.37811/cl rcm.v5i4.774 p.6368 


\begin{tabular}{|c|c|c|c|c|c|}
\hline \multicolumn{6}{|c|}{ DATOS } \\
\hline 7. Autor & Año & \multicolumn{2}{|c|}{ Nombre } & Revista & Vol. y núm. \\
\hline $\begin{array}{l}\text { Berrio, Laura } \\
\text { Cantillo, Víctor } \\
\text { Arellana, Julian }\end{array}$ & (2019) & \multicolumn{2}{|c|}{$10 \begin{array}{l}\text { STRATEGIC MODELLING } \\
\text { PASSENGER TRANSPORT } \\
\text { IN } \\
\text { WATERWAYS: THE CASE OF } \\
\text { THE MAGDALENA RIVER }\end{array}$} & $\begin{array}{c}\text { Transport } \\
\underline{\text { https://journals.vgtu.lt/index.php/Transport }} \\
\underline{\text { /article/view/8943 }}\end{array}$ & \multirow[t]{2}{*}{$34(3)$} \\
\hline \multicolumn{5}{|c|}{ CONTENIDO } & \\
\hline Tipo y Diseño & $\begin{array}{c}\text { Población y } \\
\text { Muestra }\end{array}$ & Instrumentos & Aspectos éticos & Resultados & Conclusión \\
\hline $\begin{array}{l}\text { Básica y no } \\
\text { experimental }\end{array}$ & $\begin{array}{l}7 \text { puertos del } \\
\text { río } \\
\text { Magdalena }\end{array}$ & $\begin{array}{l}\text { Análisis de } \\
\text { contenido } \\
\text { Cuestionarios }\end{array}$ & $\begin{array}{l}\text { Presentación de } \\
\text { información } \\
\text { objetiva. }\end{array}$ & $\begin{array}{l}\text { Los resultados muestran que las variables } \\
\text { de accesibilidad y las relacionadas con el } \\
\text { tamaño de la zona definen la generación y } \\
\text { distribución de viajes. Además, se encontró } \\
\text { que los usuarios de vías navegables } \\
\text { interiores en Colombia son altamente } \\
\text { sensibles a los costos. }\end{array}$ & $\begin{array}{l}\text { Los modelos propuestos } \\
\text { contribuyen a comprender } \\
\text { mejor el comportamiento de } \\
\text { los pasajeros del transporte por } \\
\text { vías navegables interiores. }\end{array}$ \\
\hline
\end{tabular}




\begin{tabular}{|c|c|c|c|c|c|}
\hline \multicolumn{6}{|c|}{ DATOS } \\
\hline 8. Autor & Año & \multicolumn{2}{|c|}{ Nombre } & Revista & Vol. y núm. \\
\hline $\begin{array}{l}\text { Shirokova, Galina } \\
\text { Ivvonen, } \\
\text { Liudmila } \\
\text { Gafforova, Elena }\end{array}$ & (2019) & \multicolumn{2}{|c|}{$\begin{array}{l}11 \text { Strategic Entrepreneurship } \\
\text { in Russia during Economic } \\
\text { Crisis }\end{array}$} & $\begin{array}{c}\begin{array}{c}\text { Foresight and STI } \\
\text { Governance }\end{array} \\
\underline{\text { http://dx.doi.org/10.17323 }} \\
\underline{22500-2597.2019 .3 .62 .76}\end{array}$ & $13(3)$ \\
\hline \multicolumn{6}{|c|}{ CONTENIDO } \\
\hline Tipo y Diseño & $\begin{array}{l}\text { Población } \\
\text { y Muestra }\end{array}$ & Instrumentos & $\begin{array}{l}\text { Aspectos } \\
\text { éticos }\end{array}$ & Resultados & Conclusión \\
\hline $\begin{array}{l}\text { Básica y no } \\
\text { experimental }\end{array}$ & 14 pymes & Encuesta & $\begin{array}{l}\text { Presentación } \\
\text { de información } \\
\text { objetiva. }\end{array}$ & $\begin{array}{lr}\text { El } & \text { componente } \\
\text { empresarial del espíritu } \\
\text { empresarial estratégico } \\
\text { está } & \text { relacionado } \\
\text { positivamente con el } \\
\text { desempeño de las PYME } \\
\text { durante la crisis } \\
\text { económica; es más }\end{array}$ & $\begin{array}{l}\text { Los datos transversales utilizados reflejan el desempeño } \\
\text { de la empresa a corto plazo. Un posible } \\
\text { El tema de investigación adicional (longitudinal) es el } \\
\text { impacto a largo plazo de la ES en el desempeño de las } \\
\text { PYMES. Segundo, } \\
\text { la principal variable dependiente utilizada en el estudio } \\
\text { fue un } \\
\text { indicador subjetivo de las actividades de las empresas, a } \\
\text { saber } \\
\text { su percepción individual por parte de los gerentes }\end{array}$ \\
\hline
\end{tabular}

Ciencia Latina Revista Científica Multidisciplinar, Ciudad de México, México. ISN 2707-2207 / ISSN 2707-2215 (en línea), julio-agosto, 2021, Volumen 5, Número 4. https://doi.org/10.37811/cl rcm.v5i4.774 p.6370 


\subsection{Estudios revisados sobre distribución de alimentos}

\begin{tabular}{|c|c|c|c|c|c|c|c|c|}
\hline \multicolumn{9}{|c|}{ DATOS } \\
\hline 9. Autor & \multicolumn{2}{|r|}{ Año } & \multicolumn{3}{|c|}{ Nombre } & \multicolumn{2}{|c|}{ Revista } & Vol. y núm. \\
\hline $\begin{array}{l}\text { Tsang, Y.P. } \\
\text { Choy, K.L. } \\
\text { Wu, C.H. } \\
\text { Ho, G.T.S. } \\
\text { Lam, H.Y. } \\
\text { Tang, Valerie }\end{array}$ & \multicolumn{2}{|c|}{ (2018) } & \multicolumn{3}{|c|}{$\begin{array}{c}\text { An intelligent model for } \\
\text { assuring food quality in } \\
\text { managing a multi- } \\
\text { temperature food } \\
\text { distribution centre }\end{array}$} & \multicolumn{2}{|c|}{$\begin{array}{l}\text { Food Control } \\
\text { https://doi.org/10.1016/j.foodcont.2018.02.030 }\end{array}$} & 90 \\
\hline \multicolumn{9}{|c|}{ CONTENIDO } \\
\hline Tipo y Diseño & \multicolumn{2}{|c|}{$\begin{array}{l}\text { Población } \\
\text { y Muestra }\end{array}$} & \multicolumn{2}{|c|}{ Instrumentos } & $\begin{array}{l}\text { Aspectos } \\
\text { éticos }\end{array}$ & \multicolumn{2}{|c|}{ Resultados } & Conclusión \\
\hline $\begin{array}{l}\text { Estudio de tipo } \\
\text { aplicada y } \\
\text { diseño } \\
\text { experimental }\end{array}$ & \multicolumn{2}{|c|}{$\begin{array}{c}\text { Un } \\
\text { sistema de } \\
\text { planificaci } \\
\text { ón de rutas } \\
\text { (IRPS) }\end{array}$} & \multicolumn{2}{|c|}{$\begin{array}{l}\text { Sensores } \\
\text { Ficha de } \\
\text { observación }\end{array}$} & $\begin{array}{l}\text { Presentación } \\
\text { de } \\
\text { información } \\
\text { objetiva. }\end{array}$ & \multicolumn{2}{|c|}{$\begin{array}{l}\text { En el marco del internet de las cosas, la } \\
\text { información ambiental se puede recopilar } \\
\text { automáticamente mediante la construcción de } \\
\text { una red de sensores inalámbricos para desarrollar } \\
\text { el monitoreo total del producto durante el proceso } \\
\text { de distribución. }\end{array}$} & $\begin{array}{l}\text { Con la ayuda de sistema de planificación de } \\
\text { rutas, se puede reducir la tasa de deterioro } \\
\text { de los alimentos durante el transporte y el } \\
\text { tiempo necesario en la planificación de rutas } \\
\text { y en la entrega de alimentos deteriorados, } \\
\text { mientras se mejora la satisfacción del } \\
\text { cliente. }\end{array}$ \\
\hline \multicolumn{9}{|c|}{ DATOS } \\
\hline \multicolumn{2}{|c|}{ 10. Autor } & \multicolumn{2}{|c|}{ Año } & \multicolumn{3}{|c|}{ Nombre } & Revista & Vol. y núm. \\
\hline \multicolumn{2}{|c|}{$\begin{array}{l}\text { Todorovic, Vladimir } \\
\text { Maslaric, Marinko } \\
\text { Bojic, Sanja } \\
\text { Jokic, Maja } \\
\text { Mircetic, Dejan } \\
\text { Nikolicic, Svetlana. }\end{array}$} & \multicolumn{2}{|c|}{$(2018)$} & & $\begin{array}{l}\text { olutions for } \\
\text { istribution in the } \\
\text { hains }\end{array}$ & $\begin{array}{l}\text { More Sustainable } \\
\text { Short Food Supply }\end{array}$ & $\begin{array}{r}\text { Sustainability } \\
\text { https://doi.org/10.3390/s }\end{array}$ & su10103481 \\
\hline
\end{tabular}




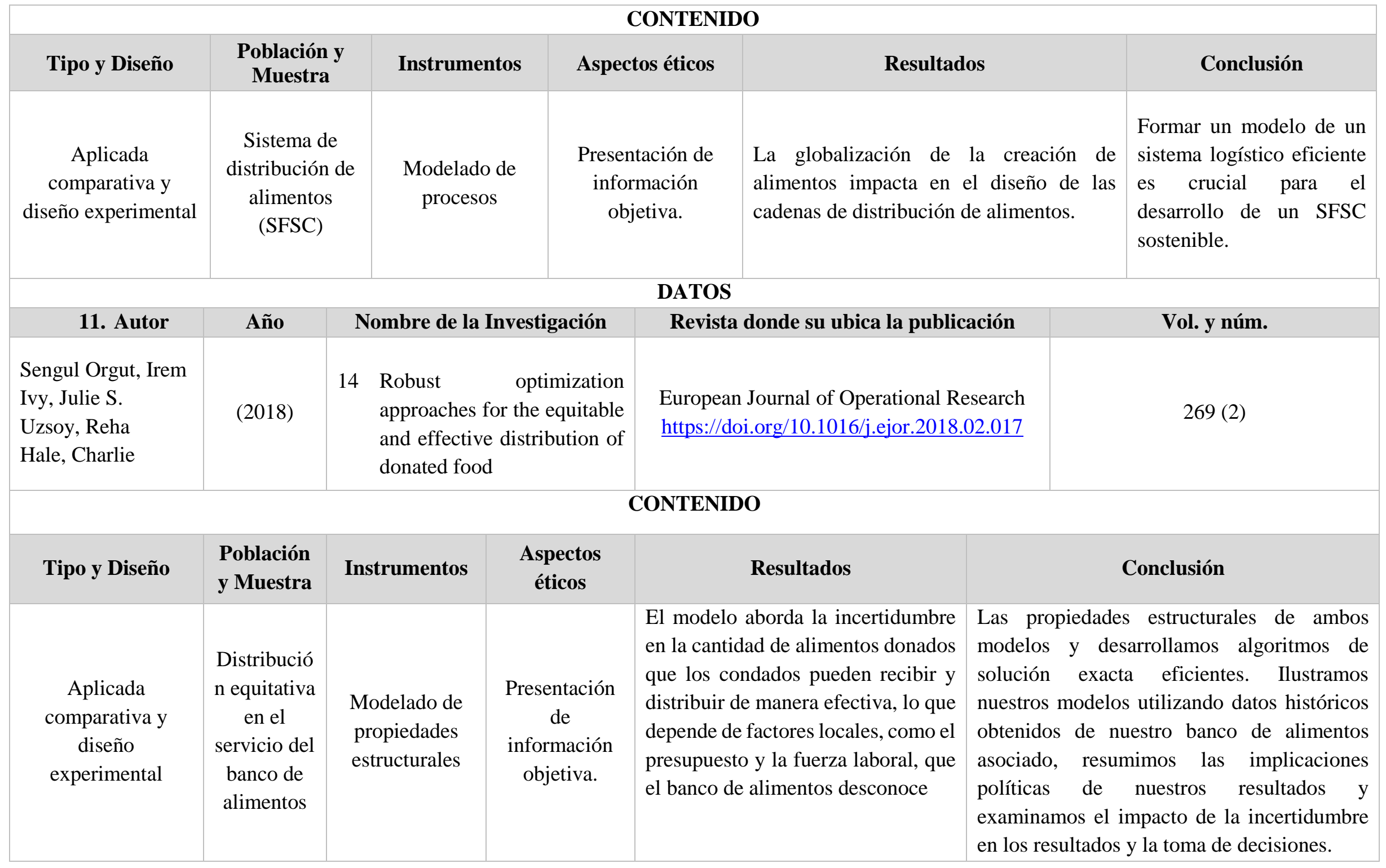

Ciencia Latina Revista Científica Multidisciplinar, Ciudad de México, México. ISN 2707-2207 / ISSN 2707-2215 (en línea), julio-agosto, 2021, Volumen 5, Número 4. https://doi.org/10.37811/cl rcm.v5i4.774 p.6372 


\begin{tabular}{|c|c|c|c|c|c|}
\hline \multicolumn{6}{|c|}{ DATOS } \\
\hline 12. Autor & Año & \multicolumn{2}{|c|}{ Nombre } & Revista & Vol. y núm. \\
\hline $\begin{array}{l}\text { Mogale, D. G. } \\
\text { Cheikhrouhou, } \\
\text { Naoufel } \\
\text { Tiwari, Manoj Kumar }\end{array}$ & (2020) & \multicolumn{2}{|c|}{$\begin{array}{l}15 \text { Modelling of sustainable } \\
\text { food grain supply chain } \\
\text { distribution system: a bi- } \\
\text { objective approach }\end{array}$} & $\begin{array}{c}\text { International Journal of Production Research } \\
\text { https://doi.org/10.1080/00207543.2019.1669840 }\end{array}$ & $58(18)$ \\
\hline \multicolumn{6}{|c|}{ CONTENIDO } \\
\hline Tipo y Diseño & $\begin{array}{c}\text { Población y } \\
\text { Muestra }\end{array}$ & Instrumentos & $\begin{array}{l}\text { Aspectos } \\
\text { éticos }\end{array}$ & Resultados & Conclusión \\
\hline $\begin{array}{l}\text { Aplicada comparativa } \\
\text { y diseño experimental }\end{array}$ & $\begin{array}{l}\text { cadena de } \\
\text { suministro de } \\
\text { granos } \\
\text { alimentarios } \\
\text { sostenibles }\end{array}$ & $\begin{array}{l}\text { Modelo de } \\
\text { toma de } \\
\text { decisiones }\end{array}$ & $\begin{array}{l}\text { Presentación } \\
\text { de } \\
\text { información } \\
\text { objetiva. }\end{array}$ & $\begin{array}{l}\text { Los resultados del análisis de sensibilidad implican } \\
\text { que los tomadores de decisiones deben establecer } \\
\text { un número suficiente de almacenes en cada estado } \\
\text { productor y consumidor manteniendo el equilibrio } \\
\text { adecuado entre los dos objetivos }\end{array}$ & $\begin{array}{l}\text { El modelo cubre varias } \\
\text { peculiaridades } \\
\text { problema, tales como } \\
\text { transporte multi escalón, } \\
\text { multitio, multimodal, } \\
\text { abastecimiento } \\
\text { distribución múltiples, } \\
\text { emisiones causadas por } \\
\text { diversos motivos, vehículos } \\
\text { capacitados heterogéneos y } \\
\text { disponibilidad limitada, y } \\
\text { almacenes capacitados. }\end{array}$ \\
\hline
\end{tabular}

Ciencia Latina Revista Científica Multidisciplinar, Ciudad de México, México. ISN 2707-2207 / ISSN 2707-2215 (en línea), julio-agosto, 2021, Volumen 5, Número 4. https://doi.org/10.37811/cl rcm.v5i4.774 p.6373 


\begin{tabular}{|c|c|c|c|c|c|}
\hline \multicolumn{6}{|c|}{ DATOS } \\
\hline 13. Autor & Año & \multicolumn{2}{|c|}{ Nombre } & Revista & Vol. y núm. \\
\hline $\begin{array}{l}\text { Davis, Kyle } \\
\text { Frankel } \\
\text { Rulli, Maria } \\
\text { Cristina } \\
\text { Seveso, Antonio } \\
\text { D’Odorico, Paolo }\end{array}$ & (2017) & \multicolumn{2}{|c|}{ 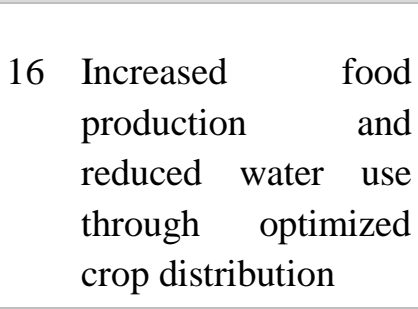 } & $\begin{array}{c}\text { Nature Geoscience } \\
\text { https://doi.org/10.1038/s41561-017-0004-5 }\end{array}$ & $10(12)$ \\
\hline \multicolumn{6}{|c|}{ CONTENIDO } \\
\hline Tipo y Diseño & $\begin{array}{l}\text { Población y } \\
\text { Muestra }\end{array}$ & $\begin{array}{l}\text { Instrume } \\
\text { ntos }\end{array}$ & $\begin{array}{l}\text { Aspectos } \\
\text { éticos }\end{array}$ & Resultados & Conclusión \\
\hline $\begin{array}{l}\text { Estudio de tipo } \\
\text { aplicada } \\
\text { comparativa y } \\
\text { diseño } \\
\text { experimental }\end{array}$ & $\begin{array}{l}14 \text { cultivos } \\
\text { alimentarios }\end{array}$ & $\begin{array}{c}\text { Modelo } \\
\text { de agua } \\
\text { de cultivo }\end{array}$ & $\begin{array}{l}\text { Presentación } \\
\text { de } \\
\text { información } \\
\text { objetiva. }\end{array}$ & $\begin{array}{l}\text { La distribución actual de cultivos en todo el } \\
\text { mundo no alcanza la máxima producción ni el } \\
\text { mínimo uso de agua. Identificamos posibles } \\
\text { configuraciones alternativas del paisaje agrícola } \\
\text { que, al remodelar la distribución global de los } \\
\text { cultivos dentro de las tierras de cultivo de } \\
\text { secano y de regadío actuales en función del } \\
\text { consumo total de agua, se alimentaría a } 825 \\
\text { millones de personas más y se reduciría el uso } \\
\text { consuntivo de agua de lluvia y de riego en un } \\
14 \% \text { y un } 12 \% \text {, respectivamente. }\end{array}$ & $\begin{array}{l}\text { El proceso de optimización no implica } \\
\text { una pérdida de diversidad de cultivos, } \\
\text { expansión de tierras de cultivo o } \\
\text { impactos en la disponibilidad de } \\
\text { nutrientes y piensos. Tampoco implica } \\
\text { necesariamente inversiones masivas en } \\
\text { tecnología moderna que en muchas } \\
\text { regiones requerirían un cambio de la } \\
\text { agricultura en pequeña escala a la } \\
\text { agricultura comercial a gran escala con } \\
\text { importantes impactos en los medios de } \\
\text { vida rurales }\end{array}$ \\
\hline
\end{tabular}

Ciencia Latina Revista Científica Multidisciplinar, Ciudad de México, México. ISN 2707-2207 / ISSN 2707-2215 (en línea), julio-agosto, 2021, Volumen 5, Número 4. https://doi.org/10.37811/cl rcm.v5i4.774 p.6374 


\begin{tabular}{|c|c|c|c|c|c|}
\hline \multicolumn{6}{|c|}{ DATOS } \\
\hline 14. Autor & Año & \multicolumn{2}{|c|}{ Nombre } & Revista & Vol. y núm. \\
\hline Hobbs, Jill E. & (2020) & \multicolumn{2}{|c|}{$\begin{array}{l}\text { Food supply chains } \\
\text { during the COVID-19 } \\
\text { pandemic }\end{array}$} & $\begin{array}{c}\text { Canadian Journal of Agricultural } \\
\text { Economics/Revue canadienne d'agroeconomie } \\
\text { https://doi.org/10.1111/cjag. } 12237\end{array}$ & $68(2)$ \\
\hline \multicolumn{6}{|c|}{ CONTENIDO } \\
\hline Tipo y Diseño & $\begin{array}{l}\text { Población } \\
\text { y Muestra }\end{array}$ & Instrumentos & $\begin{array}{l}\text { Aspectos } \\
\text { éticos }\end{array}$ & Resultados & Conclusión \\
\hline $\begin{array}{l}\text { Básica } \\
\text { Diseño no } \\
\text { experimental }\end{array}$ & 13 artículos & $\begin{array}{c}\text { Análisis } \\
\text { documental }\end{array}$ & $\begin{array}{l}\text { Presentación } \\
\quad \text { de } \\
\text { información } \\
\text { objetiva. }\end{array}$ & $\begin{array}{l}\text { La pandemia de COVID-19 tiene una serie de } \\
\text { implicaciones para las cadenas de suministro de } \\
\text { alimentos canadienses. La primera es la necesidad } \\
\text { de centrarse en mantener y mejorar la resiliencia } \\
\text { de la cadena de suministro. }\end{array}$ & $\begin{array}{l}\text { El modelo de cadena de suministro } \\
\text { justo a tiempo es eficiente y eficaz en } \\
\text { circunstancias normales. La } \\
\text { experiencia en las primeras etapas de } \\
\text { la pandemia sugiere que este modelo } \\
\text { puede ser vulnerable a } \\
\text { interrupciones a corto plazo } \\
\text { causadas por choques exógenos de } \\
\text { oferta y demanda }\end{array}$ \\
\hline
\end{tabular}




\begin{tabular}{|l|l|l|l|l|}
\hline \multicolumn{1}{|c|}{ 15. Autor } & Año & Nombre & Revista & Vol. y núm. \\
\hline $\begin{array}{l}\text { Lioutas, Evagelos } \\
\text { Charatsari, Chrysanthi }\end{array}$ & $(2020)$ & $\begin{array}{l}\text { 18 } \\
\text { Smart farming and short } \\
\text { food supply chains: Are } \\
\text { they compatible? }\end{array}$ & $\begin{array}{l}\text { Land Use Policy } \\
\text { https://doi.org/10.1016/j.landusepol.2020.104541 }\end{array}$ & 94 \\
\hline
\end{tabular}

\begin{tabular}{|c|c|c|c|c|c|}
\hline \multicolumn{6}{|c|}{ CONTENIDO } \\
\hline Tipo y Diseño & $\begin{array}{c}\text { Población y } \\
\text { Muestra }\end{array}$ & Instrumentos & $\begin{array}{l}\text { Aspectos } \\
\text { éticos }\end{array}$ & Resultados & Conclusión \\
\hline $\begin{array}{c}\text { Básica y } \\
\text { no experimental }\end{array}$ & $\begin{array}{c}16 \\
\text { agricultores y } \\
\text { consumidores }\end{array}$ & $\begin{array}{c}\text { Análisis } \\
\text { documental } \\
\text { Entrevistas } \\
\text { Cuestionario }\end{array}$ & $\begin{array}{l}\text { Presentación } \\
\text { de } \\
\text { información } \\
\text { objetiva. }\end{array}$ & $\begin{array}{l}\text { La incompatibilidad percibida es fundamental para } \\
\text { predecir esta disposición tanto para los agricultores } \\
\text { como para los consumidores. La línea cualitativa del } \\
\text { estudio descubrió la existencia de dos tipos diferentes } \\
\text { de compatibilidad. La compatibilidad real se refiere a } \\
\text { la coherencia de las tecnologías inteligentes con el } \\
\text { avance tecnológico de las granjas y las necesidades } \\
\text { cotidianas reales de los agricultores. La compatibilidad } \\
\text { simbólica se relaciona con los significados que los } \\
\text { agricultores y los consumidores atribuyen tanto a los } \\
\text { SFSC como a las tecnologías inteligentes }\end{array}$ & $\begin{array}{l}\text { Las tecnologías } \\
\text { inteligentes se } \\
\text { consideran } \\
\text { herramientas que } \\
\text { pueden conducir a una } \\
\text { convencionalización de } \\
\text { las cadenas de } \\
\text { suministro de alimentos } \\
\text { cortas, alterando así su } \\
\text { naturaleza } \\
\text { óptimamente distinta. }\end{array}$ \\
\hline
\end{tabular}

Ciencia Latina Revista Científica Multidisciplinar, Ciudad de México, México. ISN 2707-2207 / ISSN 2707-2215 (en línea), julio-agosto, 2021, Volumen 5, Número 4. https://doi.org/10.37811/cl_rcm.v5i4.774 p.6376 


\begin{tabular}{|l|c|c|c|c|}
\hline \multicolumn{1}{|c|}{ 16. Autores } & Año & Nombre & DATOS & Vovista \\
\hline $\begin{array}{l}\text { Bortolini, Marco } \\
\text { Galizia, Francesco } \\
\text { Mora, Cristina } \\
\text { Botti, Lucia } \\
\text { Rosano, Michele }\end{array}$ & (2018) & $\begin{array}{r}\text { 19 } \\
\text { Bi-objective design of fresh } \\
\text { food supply chain networks } \\
\text { with reusable and disposable } \\
\text { packaging containers }\end{array}$ & $\begin{array}{l}\text { Journal of Cleaner Production } \\
\text { https://doi.org/10.1016/j.jclepro.2018.02.231 }\end{array}$ & 184 \\
\hline
\end{tabular}

\begin{tabular}{|c|c|c|c|c|c|}
\hline \multicolumn{6}{|c|}{ CONTENIDO } \\
\hline Tipo y Diseño & $\begin{array}{c}\text { Población } \\
\text { y } \\
\text { Muestra }\end{array}$ & Instrumentos & Aspectos éticos & Resultados & Conclusión \\
\hline $\begin{array}{c}\text { Aplicado } \\
\text { Diseño no } \\
\text { experimental }\end{array}$ & $\begin{array}{l}\text { Diseño de } \\
\text { una red de } \\
\text { cadena de } \\
\text { suministro }\end{array}$ & $\begin{array}{c}\text { Análisis } \\
\text { documental }\end{array}$ & $\begin{array}{l}\text { Presentación de } \\
\text { información } \\
\text { objetiva. }\end{array}$ & $\begin{array}{l}\text { Los resultados enfatizan la conveniencia de } \\
\text { mezclar envases de empaque reutilizables y } \\
\text { desechables para alcanzar un óptimo global. } \\
47,1 \% \text { reutilizable y } 52,9 \% \text { desechable es una } \\
\text { posible configuración de buen equilibrio que } \\
\text { reduce las emisiones al tiempo que contiene el } \\
\text { aumento de costes. }\end{array}$ & $\begin{array}{l}\text { El modelo analiza la } \\
\text { minimización del costo y el } \\
\text { impacto ambiental en toda la red } \\
\text { obteniendo la frontera de Pareto } \\
\text { y el apoyo a los directores de } \\
\text { logística, los profesionales } \\
\text { industriales y los encargados de } \\
\text { la generación de políticas en el } \\
\text { diseño y la gestión de la red. }\end{array}$ \\
\hline
\end{tabular}

Ciencia Latina Revista Científica Multidisciplinar, Ciudad de México, México. ISN 2707-2207 / ISSN 2707-2215 (en línea), julio-agosto, 2021, Volumen 5, Número 4. https://doi.org/10.37811/cl rcm.v5i4.774 p.6377 


\subsection{Estudio sobre modelo estratégico y distribución de alimentos}

\begin{tabular}{|c|c|c|c|c|c|}
\hline \multicolumn{6}{|c|}{ DATOS } \\
\hline 17. Autor & Año & \multicolumn{2}{|c|}{ Nombre } & Revista & Vol. y núm. \\
\hline $\begin{array}{l}\text { Borrello, Massimiliano } \\
\text { Caracciolo, Francesco } \\
\text { Lombardi, Alessia } \\
\text { Pascucci, Stefano } \\
\text { Cembalo, Luigi }\end{array}$ & $(2017)$ & \multicolumn{2}{|c|}{$\begin{array}{l}20 \text { Consumers' Perspective on } \\
\text { Circular Economy Strategy } \\
\text { for Reducing Food Waste }\end{array}$} & $\begin{array}{c}\begin{array}{c}\text { Sustainability } \\
\text { https://doi.org/10.3390/su9 }\end{array} \\
\underline{010141}\end{array}$ & $9(1)$ \\
\hline \multicolumn{6}{|c|}{ CONTENIDO } \\
\hline Tipo y Diseño & $\begin{array}{c}\text { Población y } \\
\text { Muestra }\end{array}$ & Instrumentos & $\begin{array}{l}\text { Aspectos } \\
\text { éticos }\end{array}$ & Resultados & Conclusión \\
\hline $\begin{array}{l}\text { Aplicada y diseño } \\
\text { experimental }\end{array}$ & 1270 hogares & Encuesta & $\begin{array}{c}\text { Presentación } \\
\text { de información } \\
\text { objetiva }\end{array}$ & $\begin{array}{l}\text { Los resultados muestran un } \\
\text { retrato completo de la } \\
\text { participación potencial de } \\
\text { los consumidores en } \\
\text { circuitos cerrados } \\
\text { inspirados en los principios } \\
\text { de la economía circular }\end{array}$ & $\begin{array}{l}\text { La posición de los consumidores en el } \\
\text { modelo lineal de la cadena de } \\
\text { suministro de alimentos es } \\
\text { problemática. Sin embargo, en } \\
\text { consonancia con la literatura reciente } \\
\text { sobre la posibilidad de cambiar el } \\
\text { comportamiento del consumidor a } \\
\text { través de transformaciones socio- } \\
\text { tecnológicas }\end{array}$ \\
\hline
\end{tabular}

Ciencia Latina Revista Científica Multidisciplinar, Ciudad de México, México. ISN 2707-2207 / ISSN 2707-2215 (en línea), julio-agosto, 2021, Volumen 5, Número 4. https://doi.org/10.37811/cl rcm.v5i4.774 p.6378 


\begin{tabular}{|c|c|c|c|c|c|}
\hline \multicolumn{6}{|c|}{ DATOS } \\
\hline 18. Autor & Año & \multicolumn{2}{|c|}{ Nombre } & Revista & Vol. y núm. \\
\hline $\begin{array}{l}\text { Muller, Adrian } \\
\text { Schader, Christian } \\
\text { El-Hage Scialabba, Na } \\
\text { Brüggemann, Judith } \\
\text { Isensee, Anne } \\
\text { Erb, Karl-Heinz } \\
\text { Smith, Pete } \\
\text { Klocke, Peter } \\
\text { Leiber, Florian } \\
\text { Stolze, Matthias } \\
\text { Niggli, Urs }\end{array}$ & 2017 & \multicolumn{2}{|c|}{$\begin{array}{l}\text { Strategies for feeding the } \\
\text { world more sustainably with } \\
\text { organic agricultura }\end{array}$} & $\begin{array}{c}\text { Nature Communications } \\
\text { https://doi.org/10.1038/s41467-017-01410-w }\end{array}$ & $8(1)$ \\
\hline \multicolumn{6}{|c|}{ CONTENIDO } \\
\hline Tipo y Diseño & $\begin{array}{c}\text { Población } \\
\text { y } \\
\text { Muestra }\end{array}$ & Instrumentos & $\begin{array}{c}\text { Aspectos } \\
\text { éticos }\end{array}$ & Resultados & Conclusión \\
\hline $\begin{array}{l}\text { Aplicada y diseño } \\
\text { experimental }\end{array}$ & $\begin{array}{l}700 \\
\text { productos }\end{array}$ & Modelo Sol & $\begin{array}{c}\text { Presentación } \\
\text { de información } \\
\text { objetiva }\end{array}$ & $\begin{array}{l}\text { Los resultados principales en el documento se } \\
\text { muestran con la condición de la misma caloría, ya } \\
\text { que esto es más ilustrativo para capturar los } \\
\text { aspectos de disponibilidad de alimentos que están } \\
\text { en el centro de interés, y como los resultados } \\
\text { muestran que los escenarios que cumplen la } \\
\text { condición de la misma caloría siempre producen lo } \\
\text { mismo. o una mayor cantidad de proteína como } \\
\text { escenario de referencia y, por lo tanto, son } \\
\text { adecuados en el suministro de proteínas. }\end{array}$ & $\begin{array}{l}\text { Los sistemas alimentarios } \\
\text { sostenibles deben abordar } \\
\text { los desechos, las } \\
\text { interdependencias entre } \\
\text { cultivos, pastos y ganado } \\
\text { y el consumo humano }\end{array}$ \\
\hline
\end{tabular}




\section{DATOS}

\begin{tabular}{|c|c|c|c|c|c|}
\hline 19. Autor & Año & \multicolumn{2}{|c|}{ Nombre } & Revista & Vol. y núm. \\
\hline $\begin{array}{l}\text { Springmann, Marco } \\
\text { Wiebe, Keith } \\
\text { Mason-D'Croz, Daniel } \\
\text { Sulser, Timothy B } \\
\text { Rayner, Mike } \\
\text { Scarborough, Peter }\end{array}$ & (2018) & \multicolumn{2}{|c|}{$\begin{array}{l}22 \text { Health and nutritional aspects of } \\
\text { sustainable diet strategies and their } \\
\text { association with environmental } \\
\text { impacts: a global modelling } \\
\text { analysis with country-level detail }\end{array}$} & $\begin{array}{l}\text { The Lancet Planetary Health } \\
\text { https://doi.org/10.1016/S2542- } \\
\underline{5196(18) 30206-7}\end{array}$ & $2(10)$ \\
\hline \multicolumn{6}{|c|}{ CONTENIDO } \\
\hline Tipo y Diseño & $\begin{array}{c}\text { Población } \\
\mathbf{y} \\
\text { Muestra }\end{array}$ & Instrumentos & Aspectos éticos & Resultados & Conclusión \\
\hline $\begin{array}{l}\text { Investigación aplicada } \\
\text { y diseño experimental }\end{array}$ & 150 países & Modelado global & $\begin{array}{l}\text { Presentación de } \\
\text { información objetiva }\end{array}$ & $\begin{array}{l}\text { Seguir los objetivos ecológicos mediante la } \\
\text { sustitución de variedades de alimentos a base } \\
\text { de origen animal por variedades de alimentos } \\
\text { a base de plantas fue especialmente exitoso en } \\
\text { las naciones con salarios de las grandes ligas al } \\
\text { trabajar en niveles de suplementos y disminuir } \\
\text { la mortalidad prematura. }\end{array}$ & $\begin{array}{l}\text { En la evaluación del } \\
\text { riesgo, la mortalidad } \\
\text { prematura disminuyó } \\
\text { tanto con la disminución } \\
\text { de las fuentes de origen } \\
\text { animal como con las } \\
\text { mejoras en el equilibrio } \\
\text { energético de los } \\
\text { regímenes alimenticios. }\end{array}$ \\
\hline
\end{tabular}

Ciencia Latina Revista Científica Multidisciplinar, Ciudad de México, México. ISN 2707-2207 / ISSN 2707-2215 (en línea), julio-agosto, 2021, Volumen 5, Número 4. https://doi.org/10.37811/cl rcm.v5i4.774 p.6380 


\begin{tabular}{|c|c|c|c|c|c|}
\hline \multicolumn{6}{|c|}{ DATOS } \\
\hline 20. Autor & Año & \multicolumn{2}{|c|}{ Nombre de la Investigación } & Revista & Vol. y núm. \\
\hline $\begin{array}{l}\text { Allaoui, Hamid } \\
\text { Guo, Yuhan } \\
\text { Choudhary, Alok } \\
\text { Bloemhof, } \\
\text { Jacqueline }\end{array}$ & (2018) & \multicolumn{2}{|c|}{$23 \begin{array}{l}\text { Sustainable agro-food } \\
\text { supply chain design using } \\
\text { two-stage hybrid multi- } \\
\text { objective decision-making } \\
\text { approach }\end{array}$} & $\begin{array}{c}\text { Computers \& Operations Research } \\
\text { https://doi.org/10.1016/j.cor.2016.10.012 }\end{array}$ & 89 \\
\hline \multicolumn{6}{|c|}{$\begin{array}{lll}\text { CONTENIDO }\end{array}$} \\
\hline Tipo y Diseño & $\begin{array}{c}\text { Población y } \\
\text { Muestra }\end{array}$ & Instrumentos & $\begin{array}{l}\text { Aspectos } \\
\text { éticos }\end{array}$ & Resultados & Conclusión \\
\hline Investigación básica & $\begin{array}{l}\text { Selección de } \\
\text { socios }\end{array}$ & $\begin{array}{l}\text { Análisis de } \\
\text { documentos }\end{array}$ & $\begin{array}{l}\text { Presentaci } \\
\text { ón de } \\
\text { informaci } \\
\text { ón } \\
\text { objetiva }\end{array}$ & $\begin{array}{l}\text { Los análisis de los resultados numéricos } \\
\text { proporcionan importantes conocimientos } \\
\text { organizativos, prácticos y de políticas sobre (1) el } \\
\text { impacto de la sostenibilidad financiera y ambiental } \\
\text { en el diseño de la red de la cadena de suministro (2) } \\
\text { el análisis de compensación entre emisiones } \\
\text { ambientales, huella hídrica, implicaciones sociales y } \\
\text { costos asociados para decisión informada sobre la } \\
\text { inversión en la cadena de suministro. }\end{array}$ & $\begin{array}{l}\text { El enfoque propuesto } \\
\text { genera una frontera de } \\
\text { Pareto para ayudar a los } \\
\text { usuarios a tomar decisiones. } \\
\text { Los experimentos } \\
\text { numéricos se completan } \\
\text { utilizando datos de una } \\
\text { empresa agroalimentaria } \\
\text { para demostrar la eficiencia } \\
\text { y eficacia de la metodología } \\
\text { de solución propuesta }\end{array}$ \\
\hline
\end{tabular}

Ciencia Latina Revista Científica Multidisciplinar, Ciudad de México, México. ISN 2707-2207 / ISSN 2707-2215 (en línea), julio-agosto, 2021, Volumen 5, Número 4. https://doi.org/10.37811/cl rcm.v5i4.774 p.6381 


\section{DISCUSIÓN}

Los hallazgos encontrados en los estudios respecto a los modelos estratégicos existen procesos de toma de decisiones estratégicas que, de acuerdo a la teoría del procesamiento de información organizacional, la visión de la empresa permite argumentar la racionalidad procedimental y politización como un efecto negativo en la internacionalización de las pymes. Esto refleja en que cuando haya más internacionalización limitada las pymes pierden oportunidades en los mercados internacionales. De acuerdo a Petrou, Hadjielias, Thanos y Dimitratos (2020) quienes encontraron que la racionalidad procesal y la politización de las pymes reducen la internacionalización de las mismas. Asimismo, los autores mencionaron que un marco estratégico para un modelo rentable para la economía ayuda a las organizaciones a implementar medidas adecuadas para mantener a todos los actores involucrados en el proceso estratégico.

De acuerdo a la temática abordada Secundo, Schiuma y Jones (2019), sobre inteligencia estratégica para el desarrollo organizacional y comportamiento de los líderes de las organizaciones, evidenciaron la importancia que tiene la inteligencia estratégica de los líderes empresariales para el desarrollo y crecimiento de su organización a su cargo. Asimismo, ante esta importancia se añade el valor alto que tiene la inteligencia estratégica en el comportamiento empresarial, producto de ello se logran alcanzar más rápido los objetivos estratégicos mediante la aplicación de competencias que han sigo ganadas durante el proceso de la aplicación de la inteligencia estratégica. Como complemento, a ello se adiciona que la poca competitividad que tienen las organizaciones es resultado del bajo nivel de flexibilidad estratégica que poseen y que finalmente termina afectando negativamente al éxito organizacional. Además de que somete a una crisis de gestión institucional. Sin embargo, los diversos modelos estratégicos se pueden posicionar como un importante recurso de gestión en la organización de la gestión estratégica para mejorar la competitividad global de las organizaciones.

Al respecto, Parakhina, Godina, Boris y Ushvitsky (2017) en gestión estratégica en las universidades como factor de su competitividad global, manifestaron al igual que Secundo et al. que el problema más importante de la competitividad de las universidades rusas es la falta de flexibilidad estratégica, que afecta a todo el sistema de educación universitaria para Rusia. Complementando, la gestión estratégica es un recurso para el éxito en las organizaciones. Sin embargo, para que exista eficiencia en la gestión es muy importante que 
los líderes de las organizaciones inviertan en recursos principales para generar competitividad.

Por otro lado, en cuanto a la distribución de alimentos, Tsang (2018) demostró que la globalización de la producción de los alimentos influye en la estructura de la distribución de las cadenas de suministros de alimentos. Y ahora, por los avances de la ciencia y la tecnología y en el marco del internet de las cosas, la información ambiental se puede recopilar automáticamente mediante la construcción de una red de sensores inalámbricos para desarrollar el monitoreo total del producto durante el proceso de distribución. Esto posibilita que de acuerdo al modelo estratégico y a la aplicación e integración de la tecnología mediante el internet de las cosas (IoT) la distribución de los alimentos sea más seguras y rápidas. Ya que gracias a estos sistemas digitales es posible la planificación de rutas, mediante la cual se puede reducir la tasa de deterioro de los alimentos durante el transporte y el tiempo necesario en la planificación de rutas y en la entrega de alimentos deteriorados, mientras se mejora la satisfacción del cliente.

Por otro lado, Mogale, Cheikhrouhou y Tiwari (2019) en modelización de un sistema de distribución de la cadena de suministro de cereales alimentarios sostenible, mencionaron que la demanda de los alimentos, la degradación ambiental, las perdidas postcosecha y la escasez de recursos alientan a los tomadores de decisiones de los países en desarrollo a integrar los aspectos económicos y ambientales en el diseño de la red de distribución de los alimentos. Al respecto, Davis, Rulli, Seveso y D’Odorico (2017) en producción de alimentos mediante una distribución optimizada de los cultivos, esperan que la creciente demanda de productos agrícolas para alimentos, combustible y otros usos se satisfaga mediante una intensificación de la producción en las tierras que actualmente se encuentran bajo cultivo. Y esta intensificación implica la inversión en tecnología moderna como riego o fertilizantes, y aumentos en la frecuencia de cultivo en regiones adecuadas para múltiples temporadas de cultivo. Complementando a los autores, es importante inyectar tecnología a los procesos de cultivo como de distribución de los alimentos para que el proceso sea más eficiente.

\section{CONSLUSIÓN O CONSIDERACIONES FINALES}

En cuanto al modelo estratégico, en base a las investigaciones realizadas y analizadas se concluye que, el modelo estratégico es una herramienta poderosa en la gestión de programas sociales que permite el cumplimiento de los objetivos estratégicos de cualquier tipo de organización de una manera eficiente. Asimismo, alinea las capacidades con los 
acontecimientos de su entorno actuando de una manera proactiva sosteniendo la ventaja competitiva y el crecimiento organizacional e institucional. Además, constituye un plan diseñado para la mejora continua de la optimización de procesos claves en las que se rigen las instituciones.

En relación a la distribución de alimentos, de las investigaciones analizadas, se concluye que, la distribución de los alimentos, cumple una función principal en la generación de desarrollo para la sociedad porque permite y de una manera correcta la circulación de los productos básicos para la alimentación, sin embargo, cuanto estas carecen de control y orden, pierden eficiencia. Bajo ese sentido, recientemente, la sostenibilidad de las cadenas de suministro agroalimentarias se ha convertido en un tema de mayor interés por parte de las, las empresas, organizaciones e instituciones gubernamentales con el propósito de que está siempre cumpla su función de una manera eficiente.

En cuanto al modelo estratégico y la distribución de los alimentos, se concluye que, el modelo estratégico cumple un papel importante en la determinación de la distribución de alimentos, así mismo, hoy en día, gracias al ingenio, creatividad y disciplina del hombre, las tecnologías inteligentes son herramientas que nos conducen a una convencionalización de las cadenas de suministro de alimentos. De manera que, se logra reducir la tasa de deterioro de los alimentos durante el transporte y el tiempo necesario en la planificación de las rutas y en la entrega de los alimentos mejorando la satisfacción de los clientes.

Bajo esas conclusiones es pertinente recomendar que, al diseñar y aplicar soluciones sociales es importante insertar herramientas guiadas mediante modelos y planes estratégicos, que permitan a los encargados de tomar decisiones objetivas, centrados en los resultados, asimismo, para los operativos, poder interactuar mediante una serie de procedimientos y procesos que le permitirán lograr cumplir sus objetivos.

Se recomienda a las instituciones privadas y públicas a implementar programas sociales que satisfagan una necesidad en la población. Para ello, deben tomar en cuenta los principales elementos que permitirán tener éxito en la implementación de los programas, las cuales son; los recursos humanos, los recursos financieros y la logística. Sin esos elementos no podría ser eficiente ni tendría éxito los programas sociales. Atendiendo todo lo mencionado bajo un enfoque estratégico, que permita a las instituciones a ser reconocidas por el modo de trabajo y dirección eficiente. 


\section{LISTA DE REFERENCIAS}

Ahmadi M, Baei F, Hosseini-Amiri S, Moarefi A, Suifan TS, Sweis R. Proposing a model of manager's strategic intelligence, organization development, and entrepreneurial behavior in organizations. J Manag Dev. 2020;39(4).

Allaoui H, Guo Y, Choudhary A, Bloemhof J. Sustainable agro-food supply chain design using two-stage hybrid multi-objective decision-making approach. Comput Oper Res [Internet]. 2018;89. Available from: https://doi.org/10.1016/j.cor.2016.10.012

Berrio L, Cantillo V, Arellana J. STRATEGIC MODELLING OF PASSENGER TRANSPORT IN WATERWAYS: THE CASE OF THE MAGDALENA RIVER. Transport [Internet]. 2019;34(3). Available from: https://journals.vgtu.lt/index.php/Transport/article/view/8943

Borrello M, Caracciolo F, Lombardi A, Pascucci S, Cembalo L. Consumers' Perspective on Circular Economy Strategy for Reducing Food Waste. Sustainability [Internet]. 2017;9(1). Available from: https://doi.org/10.3390/su9010141

Bortolini M, Galizia F, Mora C, Botti L, Rosano M. Bi-objective design of fresh food supply chain networks with reusable and disposable packaging containers. J Clean Prod [Internet]. 2018;184. Available from: https://doi.org/10.1016/j.jclepro.2018.02.231

Davis KF, Rulli MC, Seveso A, D'Odorico P. Increased food production and reduced water use through optimized crop distribution. Nat Geosci [Internet]. 2017;10(12). Available from: https://doi.org/10.1038/s41561-017-0004-5

Guraou C, Duquesnois F, Catanzaro A. Entrepreneurial orientation, strategic processes and SMEs' performance in a turbulent environment: an investigation of the LanguedocRoussillon wine sector. Int J Entrep Small Bus [Internet]. 2017;32(1/2). Available from: https://doi.org/10.1504/IJESB.2017.085991

Hobbs JE. Food supply chains during the COVID-19 pandemic. Can J Agric Econ Can d'agroeconomie [Internet]. 2020;68(2). Available from: https://doi.org/10.1111/cjag.12237

Kumar V. A strategic framework for a profitable business model in the sharing economy. Ind Mark Manag [Internet]. 2018;69. Available from: https://doi.org/10.1016/j.indmarman.2017.08.021

Lioutas E, Charatsari C. Smart farming and short food supply chains: Are they compatible? Land use policy. 2020;94. 
Mogale DG, Cheikhrouhou N, Tiwari MK. Modelling of sustainable food grain supply chain distribution system: a bi-objective approach. Int J Prod Res. 2020;58(18).

Muller A, Schader C, El-Hage Scialabba N, Brüggemann J, Isensee A, Erb K-H, et al. Strategies for feeding the world more sustainably with organic agricultura. Nat Commun. 2017;8(1).

Parakhina V, Godina O, Boris O, Ushvitsky L. Strategic management in universities as a factor of their global competitiveness. Int J Educ Manag [Internet]. 2017;31(1). Available from: https://doi.org/10.1108/IJEM-03-2016-0053

Petrou AP, Hadjielias E, Thanos IC, Dimitratos P. Strategic decision-making processes, international environmental munificence and the accelerated internationalization of SMEs. Int Bus Rev [Internet]. 2020;25(5). Available from: https://doi.org/10.1016/j.ibusrev.2020.101735

Secundo G, Schiuma G, Jones P. Strategic knowledge management models and tools for entrepreneurial universities. Manag Decis [Internet]. 2019;57(12). Available from: https://doi.org/10.1108/MD-12-2019-027

Sengul Orgut I, Ivy JS, Uzsoy R, Hale C. Robust optimization approaches for the equitable and effective distribution of donated food. Eur J Oper Res [Internet]. 2018;269(2). Available from: https://doi.org/10.1016/j.ejor.2018.02.017

Shirokova G, Ivvonen L, Gafforova E. Strategic Entrepreneurship in Russia during Economic Crisis. Foresight STI Gov [Internet]. 2019;13(3). Available from: http://dx.doi.org/10.17323/2500-2597.2019.3.62.76

Springmann M, Wiebe K, Mason-D’Croz D, Sulser TB, Rayner M, Scarborough P. Health and nutritional aspects of sustainable diet strategies and their association with environmental impacts: a global modelling analysis with country-level detail. Lancet Planet Heal [Internet]. 2018;2(10). Available from: https://doi.org/10.1016/S25425196(18)30206-7

Todorovic V, Maslaric M, Bojic S, Jokic M, Mircetic D, Nikolicic S. Solutions for More Sustainable Distribution in the Short Food Supply Chains. Sustainability [Internet]. 2018;10(10). Available from: https://doi.org/10.3390/su10103481

Tsang YP, Choy KL, Wu CH, Ho GTS, Lam HY, Tang V. An intelligent model for assuring food quality in managing a multi-temperature food distribution centre. Food Control [Internet]. 2018;90. Available from: https://doi.org/10.1016/j.foodcont.2018.02.030 
